\title{
Signal Enhancement of the Central-Transition Signal in Solid-State NMR of Quadrupolar Nuclei with Spin $I=5 / 2$ Using Fast Amplitude-Modulated (FAM) Pulse Trains: A Computational Study
}

\author{
Thomas Bräuniger*
}

Institute of Physics, University of Halle, Betty-Heimann-Str. 7, 06120 Halle, Germany

\begin{abstract}
In solid-state NMR of quadrupolar nuclei with half-integer spin $I$, a variety of fast amplitude-modulated (FAM) pulse trains have been used to enhance the intensity of the central-transition signal, by transferring spin population from the satellite transitions. Here, we describe the results of extensive numerical calculations using the Simpson program package to evaluate and compare the signal enhancing performance of FAM-I, SW( $\tau$ )-FAM, and $\mathrm{SW}(1 / \tau)$-FAM sequences under both static and MAS conditions for nuclei with $I=5 / 2$. The comparative calculations were performed for a two-dimensional grid of the quadrupolar interaction parameters, namely $\chi=0.5 \ldots 4 \mathrm{MHz}, \eta=0 \ldots 1$, and a range of MAS spinning speeds, i.e. $0 \mathrm{kHz}$ (static), $2.5 \mathrm{kHz}, 5 \mathrm{kHz}$ and $10 \mathrm{kHz}$. In agreement with previously published experimental data, the best signal enhancement of static spectra is delivered by the $\mathrm{SW}(1 / \tau)$-FAM sequence, whereas under MAS conditions, $\operatorname{SW}(\tau)$-FAM and FAM-I give better results.
\end{abstract}

Keywords: Solid-state NMR, quadrupolar nuclei, sensitivity enhancement, SW-FAM.

\section{INTRODUCTION}

Most atomic nuclei observable by nuclear magnetic resonance (NMR) spectroscopy have half-integer spin $I>$ $1 / 2$, and thus possess a quadrupolar moment $[1,2]$. Such nuclei also constitute the majority of spins observable by NMR spectroscopy in technologically important materials such as minerals, ceramics, catalysts and glasses [3], e.g. ${ }^{7} \mathrm{Li}$, ${ }^{11} \mathrm{~B},{ }^{23} \mathrm{Na}$ with $\mathrm{I}=3 / 2,{ }^{17} \mathrm{O},{ }^{25} \mathrm{Mg},{ }^{27} \mathrm{Al}$ with $I=5 / 2$, and ${ }^{43} \mathrm{Ca}$, ${ }^{45} \mathrm{Sc},{ }^{51} \mathrm{~V}$ with $I=7 / 2$. In most cases, the solid-state NMR characterisation of inorganic materials is restricted to observing the position and shape of the second-order quadrupolar broadened central-transition (CT) peak. In the absence of spectral overlap or parameter distributions, the chemical shift and the quadrupolar interaction parameters may be determined from the characteristic 'second-order shape' of the CT $[1,2]$, and these parameters may then be correlated to structure and properties of the materials. However, even with magic-angle-spinning (MAS) [4] for partial averaging of the second-order broadening of the spectral lines, sensitivity problems persist for numerous nuclei, caused by small gyromagnetic ratios, low natural abundance, strong quadrupolar interaction or by any combination of these factors.

For one-dimensional spectra, the intensity of the centraltransition (CT) signal can be increased by transferring spin population from the satellite transitions (ST). Complete inversion of the STs of a spin $I$ leads to an enhancement of the CT intensity by a factor of $2 I$, while complete saturation of the STs results in an improvement factor of $I+1 / 2$, as schematically shown in Fig. (1a). Apparently, the first

*Address correspondence to this author at the Institute of Physics, University of Halle, Betty-Heimann-Str. 7, 06120 Halle, Germany; Fax: +49 345 5527161; E-mail: thomas.braeuniger@physik.uni-halle.de experimental evidence showing enhancement of the $\mathrm{CT}$ as a result of manipulation of the STs was published by Pound already in 1950 [5]. Since the re-introduction of this principle by Vega and Naor in 1981 [6], and in a series of detailed papers by Haase et al. [7-9], several experimental techniques have been put forward to effect the necessary manipulations of the STs [10-25]. Among these techniques are double frequency sweeps (DFS) [10-13], the application of hyperbolic secant pulses (HS) [26] to quadrupolar nuclei [1416], and the use of pulse trains with alternating phases, designated either "rotor assisted population transfer" (RAPT/FSG-RAPT) [17-19] or "fast-amplitude modulated" (FAM) pulse trains [20-25]. More details about these methods and their development may be found in the introductions of Refs. [16, 24].

In this paper, we will investigate the signal enhancement produced by different varieties of FAM pulse trains, with particular focus on the comparative performance of the respective FAM trains when transitioning from static to magic-angle spinning (MAS) conditions. As a model nucleus, ${ }^{27} \mathrm{Al}$ with $I=5 / 2$ is chosen, to facilitate comparison to previously published results $[20,22,24]$. FAM pulse trains act on the spin system by generating sidebands of the RF carrier (=Larmor) frequency $\omega_{0}$ using a series of pulse pairs, with each of the pairs consisting of two RF pulses with a $180^{\circ}$ phase shift between them. The simplest FAM pulse train ("FAM-I") is formed by a series of pulse pairs of uniform duration $\tau_{p}$, separated by constant interpulse delays (usually of duration $\tau_{p}$ as well), and repeated $N$ times. Such uniform pulse pairs produce strong main sidebands at $\omega_{0}+\omega$ and $\omega_{0}-$ $\omega$, with $\omega$ determined by the pulse durations, $\omega=2 \pi /\left(4 \tau_{p}\right)$, plus higher-order sidebands (the next being at $\omega_{0} \pm 3 \omega$ ), which are however much weaker [24]. As shown in Fig. (1b), (I -1/2) modulation frequencies are needed to traverse all satellite transitions of a nucleus with half-integer spin $I$ for a 

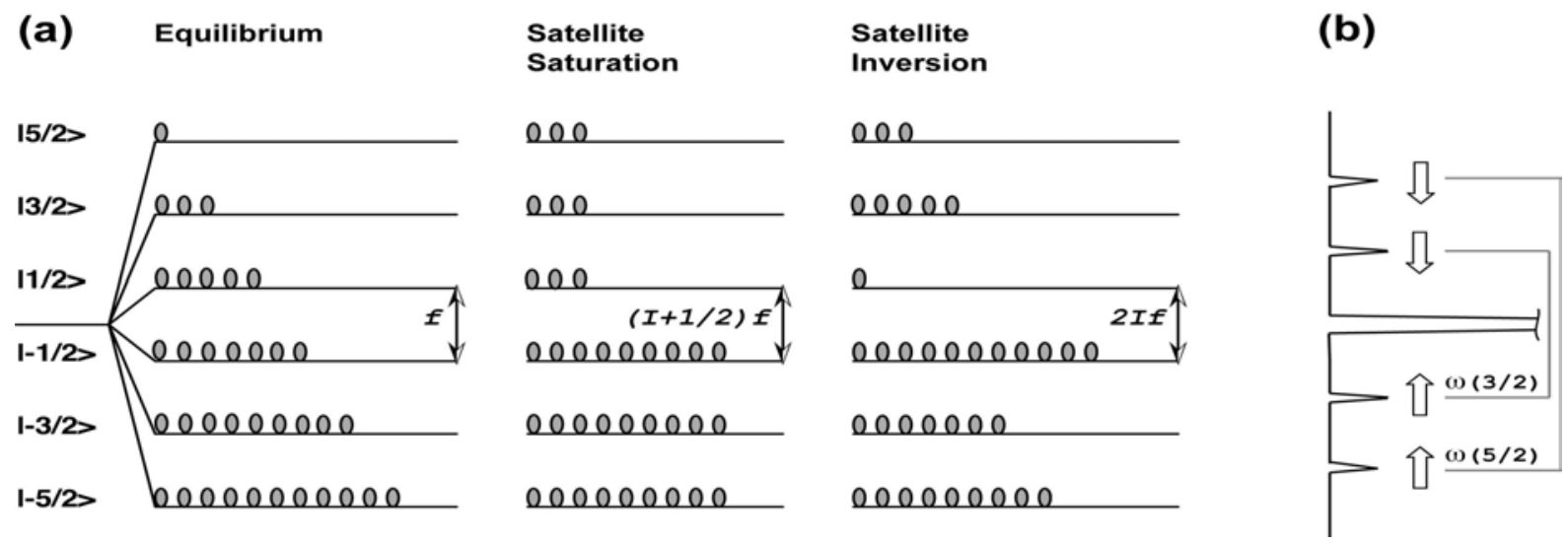

Fig. (1). (a) Schematic (exaggerated) spin population distribution for an $I=5 / 2$ nucleus in an external magnetic field. The spectral intensity for the central transition $(m=-1 / 2 \rightarrow+1 / 2)$ is proportional to the population difference $f$. In the case of saturation of the satellite transitions, the achieved enhancement factor for the central-transition signal is $(I+1 / 2)$, while for selective inversion of the satellite transitions (progressing from the outer to the inner ones), the enhancement factor is $2 I$. (b) Schematic representation of a single-crystal NMR spectrum of an $I=5 / 2$ nucleus, with central-transition and satellite-transition peaks. For manipulation of the spin level populations, the frequencies of the applied RF modulations need to be matched to the positions of the satellite peaks, $\omega(3 / 2)$ and $\omega(5 / 2)$. The arrows indicate the direction of the frequency sweep applied in DFS [10] and SW-FAM [22].

single crystal spectrum. In the case of $I=5 / 2$, the two necessary modulation frequencies may be generated by appropriate adjustment of the pulse durations of two consecutive FAM-I trains, as schematically shown in Fig. (2). The majority of compounds examined by NMR are however not available in single crystal form, but as polycrystalline (powder) samples. In powder samples, the RF effects on the spins vary for different crystallites, since the resonance frequency of a quadrupolar nucleus depends on the orientation of the principal axis system of the electric field gradient tensor relative to the external magnetic field. Therefore, the resonance frequencies of the individual crystallites are statistically oriented over all orientations, and superimpose to produce a characteristic "powder pattern" lineshape. For acquisition of static spectra, and/or when the nucleus to be observed has more than one satellite transition (as with spin-5/2), it is therefore beneficial to replace the individual modulation frequencies of FAM-I pulse trains by a range of frequencies. This can be accomplished by using frequency-swept FAM trains ("SW-FAM"), where the durations of the RF pulse pairs are changed continuously within the pulse train, as shown schematically in Fig. (2b). The sweep of modulation frequency may be carried out in different ways. In the original SW-FAM scheme [22], constant time increments $\Delta$ are added to the duration of both the RF pulses and interpulse delays $\tau_{p}$. Thus, a pulse train starting with a modulation period of $\tau^{0}=4 \tau_{p}^{0}$ for the first FAM pulse pair, would finish with a modulation period $\tau^{\mathrm{N}-1}=4 \tau_{p}^{N-1}$ after the execution of $N$ pulse pairs, with $\tau_{p}^{N-1}=\tau_{p}^{0}+(\mathrm{N}-1) \Delta$. This produces a curved distribution of frequencies, as shown in Fig. (2c) (filled circles). Because the modulation periods $\tau^{i}$ for the $i$ th pulse pair are being incremented linearly, such pulse trains are designated $\mathrm{SW}(\tau)$ FAM. An alternative approach is to generate a frequency distribution $\left(1 / \tau^{i}\right)$ which is linear with respect to the pulse pair progression. The resulting pulse train (see Fig. (2c), open circles) is referred to as $\mathrm{SW}(1 / \tau)$-FAM, and has been shown to give superior enhancement for static samples compared to $\mathrm{SW}(\tau)$-FAM because of the even distribution of modulation frequencies [24, 25].

(a)

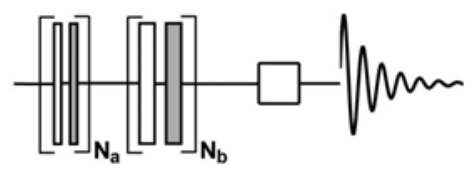

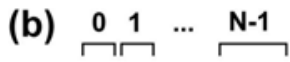

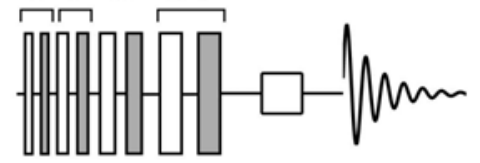

(c)

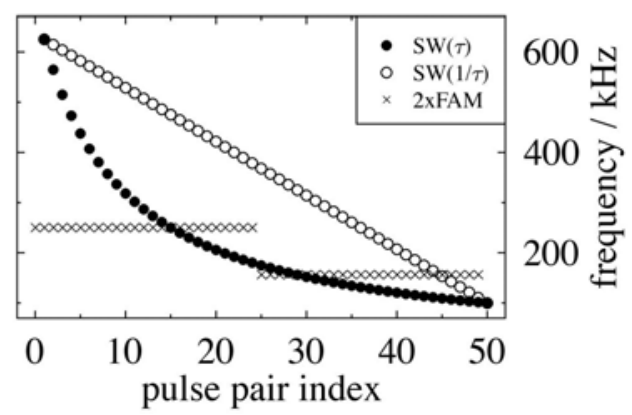

Fig. (2). (a) Schematic representation of two FAM-I pulse trains with $N_{a}$ and $N_{b}$ pulse pairs respectively, followed by a read pulse and the resulting FID. The alternating white and grey colours of the RF pulses signify the $180^{\circ}$ phase shift. (b) Schematic representation of the RF pulse progression in an SW-FAM pulse train with $N$ pulse pairs sweeping from higher to lower modulation frequencies (with the pulse pair index indicated above the pulses), plus read pulse and the resulting FID. (c) Modulation frequencies generated by a doubleblock FAM-I pulse train ( $\left.\tau_{p}^{a}=1.0 \mu \mathrm{s}, \tau_{p}^{b}=1.6 \mu \mathrm{s}, N_{a}=N_{b}=25\right)$, $\mathrm{SW}(\tau)$-FAM and SW $(1 / \tau)$-FAM pulse trains $\left(\tau_{p}^{0}=0.4 \mu \mathrm{s}, \tau_{p}^{49}=2.5\right.$ $\mu$ s, i.e. sweep width $=525 \mathrm{kHz}$ ), with $N=50$ pulse pairs. The necessary formulae to compute the pulse trains for both $\mathrm{SW}(\tau)$-and $\mathrm{SW}(1 / \tau)$-FAM may be found in Ref. [24]. 
The situation changes with the application of magic-angle spinning (MAS) to a powder sample, because the rotor motion imposes a time dependence on the resonance frequencies of the individual crystallites. The performance of FAM-I pulse trains benefits from that, because MAS spreads out the effect of the "monochromatic" RF (the acronym RAPT [17-19], "rotor assisted population transfer", refers to this process). Whereas the considerable enhancement of central-transition MAS spectra by application of FAM-I trains has been extensively documented before [17-23], fewer data exist about the signal enhancement obtainable by $\mathrm{SW}(\tau)$-and $\mathrm{SW}(1 / \tau)$-FAM. The enhancement generated by $\mathrm{SW}(\tau)$-FAM under MAS has been shown to be in about the same range as that obtained from one or several FAM-I trains [22, 23, 25]. However, the performance of $\mathrm{SW}(1 / \tau)$-FAM, which is the best choice for static conditions, has been recently shown to degrade with the onset of MAS [25]. It is the purpose of this paper to explore the comparative signal enhancement potential of the FAM, SW $(\tau)$-FAM, and $\mathrm{SW}(1 / \tau)$-FAM sequences under MAS in more detail. To this end, extensive numerical calculations have been carried out using the Simpson package [27]. To assess the feasible signal enhancement systematically for a range of quadrupolar coupling constants $\chi=\left(e^{2} q Q\right) / h$ and asymmetry parameters $\eta$, the calculations were performed over a grid with $\chi=0.5 \ldots 4$ $\mathrm{MHz}($ step $0.5 \mathrm{MHz})$ and $\eta=0 \ldots 1$ (step 0.25), using ${ }^{27} \mathrm{Al}(I$ $=5 / 2$ ) as a model nucleus. Evaluation of the results show that the enhancement factors available with $\mathrm{SW}(1 / \tau)$-FAM indeed slightly suffer with the onset of MAS, whereas SW $(\tau)$-FAM and FAM-I tend to perform very well also under spinning conditions.

\section{NUMERICAL SIMULATIONS}

The static ${ }^{27} \mathrm{Al}$ central-transition signal was calculated considering 28656 powder orientations according to the $\mathrm{ZCW}$ scheme [27], whereas $4180 \mathrm{ZCW}$ orientations plus $10 \gamma$ angles for each orientation were used for calculation of the MAS spectra. The central-transition RF nutation frequencies used for preceding FAM train and single read pulse were $v_{\text {nut }}(\mathrm{FAM})=60 \mathrm{kHz}$, and $v_{\text {nut }}(\mathrm{read})=30 \mathrm{kHz}$, respectively. The corresponding Simpson [27] input files are available from the author upon request.

The signal intensities of both the FAM-enhanced $\left(I_{F A M}\right)$ and the single-pulse spectrum $\left(I_{\mathrm{S} P}\right)$ were calculated by summing up the intensities of all calculated spectral points, $\Sigma_{\mathrm{i}}$ $I\left(v_{i}\right)$, with $I=1 \cdots 2048$. The obtained enhancement is then expressed by a factor which is the ratio between these intensities, $F[\mathrm{FAM}]=I_{F A M} / I_{\mathrm{S} P}$. To quantify the lineshape differences produced by the numerical calculations, the intensities of both the FAM-enhanced and the single-pulse spectrum were normalised to a common factor $C$, such that $I^{C}\left(v_{i}\right)=\left[C / \sum_{\mathrm{i}} I\left(v_{i}\right)\right] \cdot I\left(v_{i}\right)$, for every intensity point in the spectrum. Then, a root-mean-square variation between the spectra was computed as r.m.s. $=\sqrt{\sum_{i}\left(I_{F A M}^{C}\left(v_{i}\right)-I_{S P}^{C}\left(v_{i}\right)\right)^{2}}$, and $C$ being chosen such as to produce convenient numbers for the r.m.s. values of all investigated spectra.

\section{RESULTS AND DISCUSSION}

The signal enhancement factors obtained with the respective FAM pulse trains were optimised under static conditions for the midpoint of the investigated grid, i.e. for $\chi$ $=2.0 \mathrm{MHz}$ and $\eta=0.5$. In addition to the obvious criterion of enhanced intensity, the reproduction of the correct line shape is also important. The optimised pulse trains should therefore deliver a good compromise between intensity enhancement and lineshape quality. SW-FAM pulse trains can be optimised by varying 4 parameters: starting value of the modulation frequency $\left(\tau_{p}^{0}\right)$, width of frequency sweep $\left(\tau_{p}^{N-1}\right)$, rate of sweep (number of pulse pairs $N$ ), and RF field strength. These parameters also determine the adiabaticity of the SW-FAM sequence: the RF sweep is said to traverse a transition "adiabatically" when changes in the frequency are slow enough for the spin system to follow the effective field [28]. The adiabaticity of RF sweeps for multi-level systems such as quadrupolar nuclei with half-integer spin is a complex problem [13], and will not be discussed in detail here. However, slight deviations of the FAM-amplified spectra from the correct line shape (as defined by the single-pulse spectra) observed in some of the simulated spectra may possibly be assigned to a breach of the adiabaticity condition, as the duration of the FAM trains used here is generally quite short.

The FAM trains producing the spectra with the best compromise between enhancement and line shape are those depicted in Fig. (2c), with the pulse train parameters listed in the figure caption. The spectra resulting from application of these FAM trains under both static and $10 \mathrm{kHz}$ MAS conditions are shown in Fig. (3). The performance of the thus optimised pulse trains was subsequently tested over the entire $\{\chi, \eta\}$ grid, without further optimisation. Enhancement and line shape quality were evaluated for static conditions, and for MAS with $2.5 \mathrm{kHz}, 5 \mathrm{kHz}$ and $10 \mathrm{kHz}$ spinning speed. To illustrate the results of these numerical simulations, 3dimensional grids were constructed to display the obtained enhancement factors (Fig. (4)) and the induced deviations from the ideal line shape (Fig. (5)) under both static and 10 $\mathrm{kHz}$ MAS conditions.

When comparing the enhancement factors for static spectra (Fig. (4)), it can be seen that the spin population transfer induced by the double-block FAM-I train is not very efficient, with the enhancement factor not exceeding 1.5 anywhere on the grid, and the grid average being just 1.277. In contrast, both SW-FAM sequences are capable of delivering enhancement factors close to the saturation value of $I+1 / 2=3$ for some parts of the grid. When averaging over the entire grid, the $\mathrm{SW}(1 / \tau)$ train outperforms the $\mathrm{SW}(\tau)$ sequence by 2.375 to 2.178 . These performance trends have also been confirmed by experimental results [24]. The picture is very different for the enhancements attainable under 10 kHz MAS: here SW $(\tau)$-FAM with an average enhancement of 2.246 and double-block FAM-I with 2.134 perform better than $\mathrm{SW}(1 / \tau)$-FAM with an average of 2.028 , which is again similar to what was found by experiment previously [22]. It therefore seems that the $\mathrm{SW}(\tau)$-FAM sequence constitutes a good compromise, performing fairly well under both static and MAS conditions. In Fig. (2c), it can be seen that the curved frequency distribution of $\mathrm{SW}(\tau)$-FAM leads to a "bunching" in the region of lower frequencies, in fact in about the same region where the optimal modulation frequency of the second FAM-I block is located. The improved 
$\operatorname{SW}(1 / \tau)$-FAM $\quad S W(\tau)$-FAM
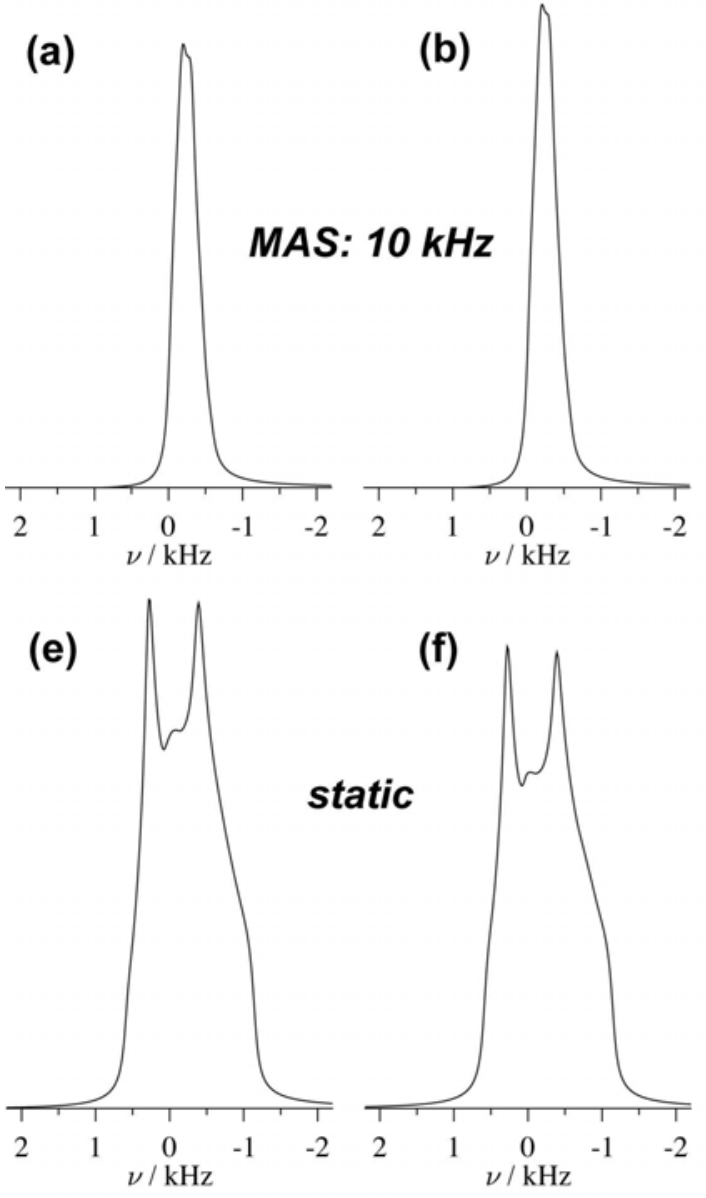

(f)
2xFAM-I

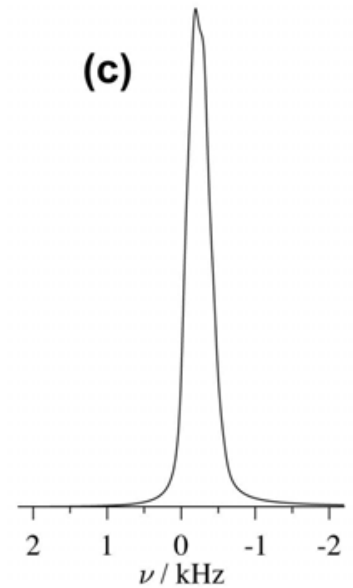

(g)

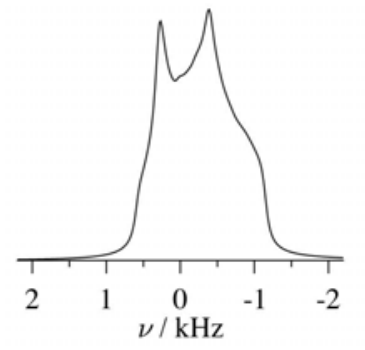

\section{single pulse}

(d)

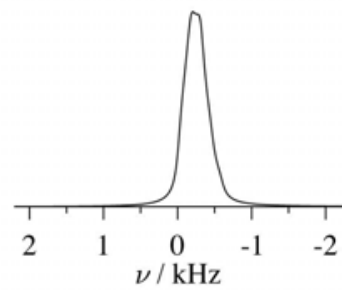

(h)

Fig. (3). ${ }^{27} \mathrm{Al}$ NMR spectra obtained by Simpson [27] calculations for the quadrupolar parameters $\chi=2.0 \mathrm{MHz}$ and $\eta=0.5$. Enhancement of the central-transition signal by spin population transfer from the satellite transitions was affected for both static and MAS spectra by applying SW $(1 / \tau)$-FAM (a,e), SW $(\tau)$-FAM (b,f), and double-block FAM-I (c,g) pulse trains prior to the single read pulse. Single pulse spectra are shown in (d) and (h). The properties of the respective FAM pulse trains are listed in the caption of Fig. (2c). The obtained enhancement factors, $F$ [pulse train], and the root-mean-square variation rms from the single-pulse spectrum (see text for details) are the following:

(a) $10 \mathrm{kHz}$ MAS spectrum with $F[\mathrm{SW}(1 / \tau)]=2.25$, and $\mathrm{rms}=0.40$.

(b) $10 \mathrm{kHz}$ MAS spectrum with $F[\mathrm{SW}(\tau)]=2.46$, and $r m s=0.17$.

(c) $10 \mathrm{kHz}$ MAS spectrum with $F[2 \times \mathrm{FAM}-\mathrm{I}]=2.48$, and $r m s=0.54$.

(e) Static spectrum with $F[\mathrm{SW}(1 / \tau)]=2.64$, and $r m s=0.94$.

(f) Static spectrum with $F[\mathrm{SW}(\tau)]=2.46$, and $r m s=0.80$.

(g) Static spectrum with $F[2 \times \mathrm{FAM}-\mathrm{I}]=1.37$, and $\mathrm{rms}=0.45$.

performance of $\mathrm{SW}(\tau)$-FAM over $\mathrm{SW}(1 / \tau)$-FAM under MAS may thus be due to its similarity to a FAM-I distribution in the last part of the pulse train, although the exact nature of the transfer process needs to be studied in more detail.

Looking at the deviation of the enhanced spectra from the single-pulse spectrum (Fig. (5)), it can be seen that the the r.m.s. variation of the static line shape is comparatively small for the FAM-I trains, which is due to the fact that the spectra are not much affected by FAM-I at all. The average r.m.s. line shape variation for static spectra is practically identical for both SW-FAM sequences, and overall larger than the one observed for the FAM-I train. The relatively large line shape deviation occuring for enhancement of static spectra reflects the difficulty to uniformly affect all powder crystallites in the absence of MAS averaging. Similar line shape problems have been encountered for static ${ }^{27} \mathrm{Al}$-spectra enhanced by hyperbolic secant (HS) pulses [15]. For all sequences in Fig. (5), the observed line shape variations are much smaller under $10 \mathrm{kHz}$ MAS. This is again a consequence of the fact that the fast rotor motion spreads out the effects of the applied RF. For static samples, the spin population transfer is solely mediated by the RF -if some crystallites are left unaffected, changes in the enhanced line shape will show up. Under MAS, however, different degrees of saturation and inversion are distributed randomly over all orientations of the sphere, which has been shown by Iuga and Kentgens [13] by a detailed analysis of the combined effect of DFS and MAS on powder samples. This statistic distribution results in a more faithful reproduction of the line shape. 
2xFAM-I

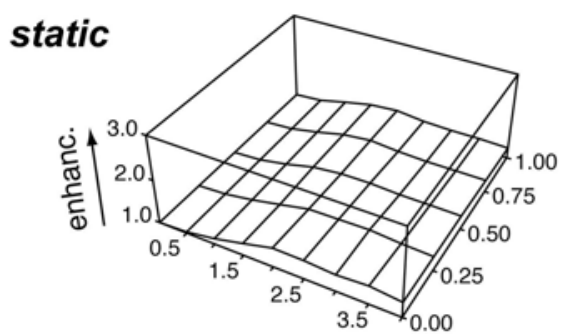

avg enhanc.: $\quad 1.277$
$\operatorname{SW}(\tau)$-FAM

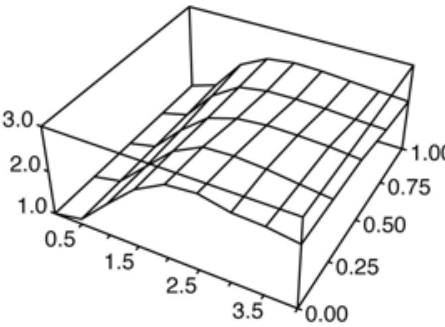

2.178
$\operatorname{SW}(1 / \tau)$-FAM

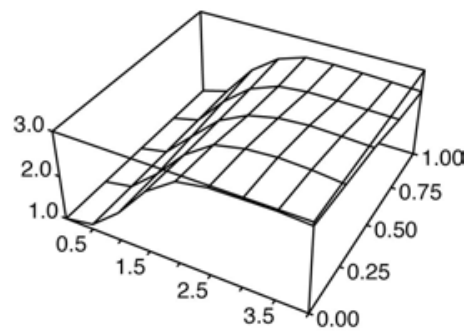

2.375

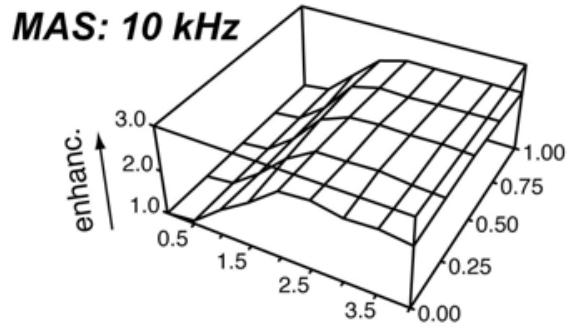

avg enhanc.: $\quad 2.134$

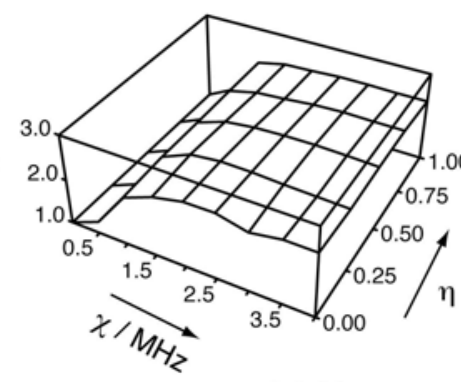

2.246

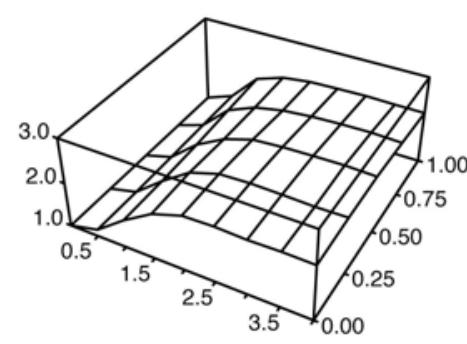

2.028

Fig. (4). Numerical simulation of central-transition signal enhancement of ${ }^{27}$ Al NMR spectra by FAM sequences: Signal enhancement performance (relative to the intensity of the single-pulse spectrum) over a grid of $\chi=0 \ldots 4 \mathrm{MHz}($ step $0.5 \mathrm{MHz})$ and $\eta=0 \ldots 1$ (step 0.25). The pulse train parameters used for FAM-I (left), SW $(\tau)$-FAM (centre) and SW $(1 / \tau)$-FAM (right) are those specified in Fig. (2c). Listed under the grids are the average enhancement factors calculated for $\chi=0.5 \ldots 4 \mathrm{MHz}$ (no enhancement is expected for $\chi=0 \mathrm{MHz}$ ) and $\eta=0 \ldots$. Top: Static spectra. Bottom: $10 \mathrm{kHz}$ MAS spectra.

2xFAM-I

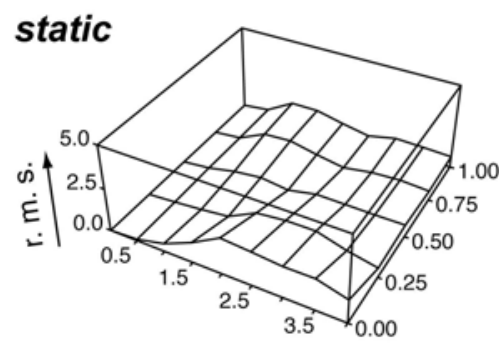

avg r.m.s.: $\quad 0.821$

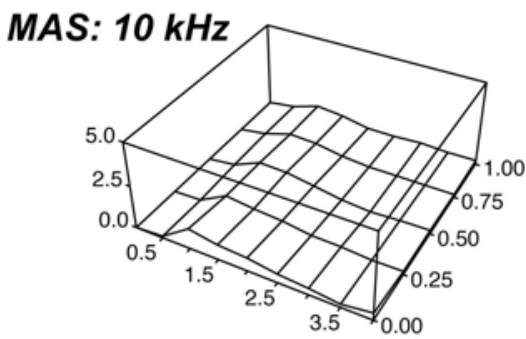

avg r.m.s.: $\quad 0.410$
$\operatorname{SW}(\tau)-F A M$

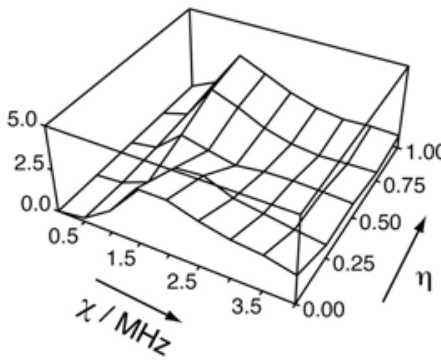

1.341

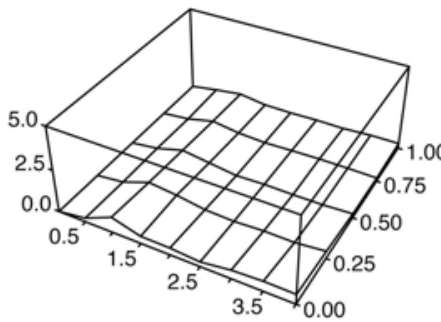

0.337

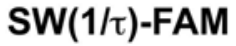

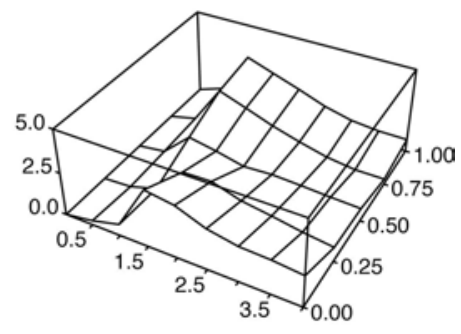

1.348

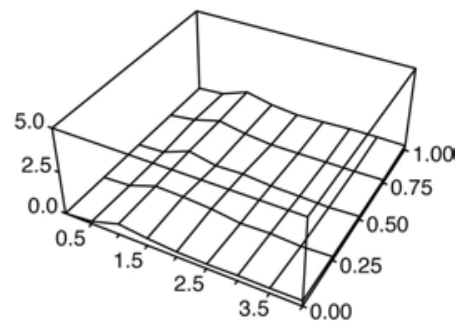

0.307

Fig. (5). Numerical simulation of central-transition signal enhancement of ${ }^{27} \mathrm{Al}$ NMR spectra by FAM sequences: r.m.s. variation of the line shape from single-pulse spectrum (see text for details) over a grid of $\chi=0 \ldots 4 \mathrm{MHz}$ (step $0.5 \mathrm{MHz}$ ) and $\eta=0 \ldots 1$ (step 0.25 ). The pulse train parameters used for FAM-I (left), SW $(\tau)$-FAM (centre) and SW $(1 / \tau)$-FAM (right) are those specified in Fig. (2c). Listed under the grids are the average r.m.s. variation calculated for $\chi=0.5 \ldots 4 \mathrm{MHz}$ (no variation is expected for $\chi=0 \mathrm{MHz}$ ) and $\eta=0 \ldots 1$. Top: Static spectra. Bottom: $10 \mathrm{kHz}$ MAS spectra. 
optimised point

(a)

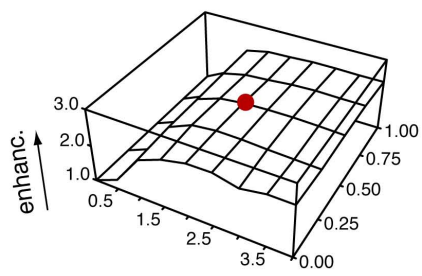

(b)

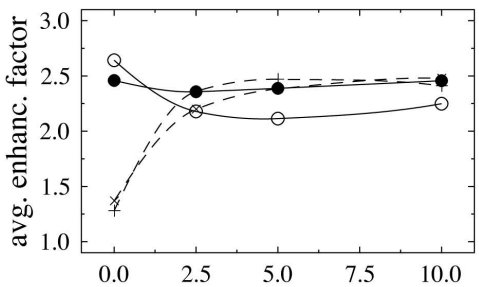

(c)

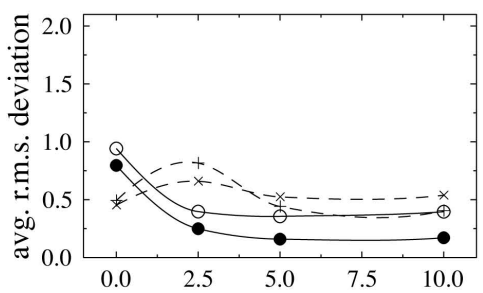

average line
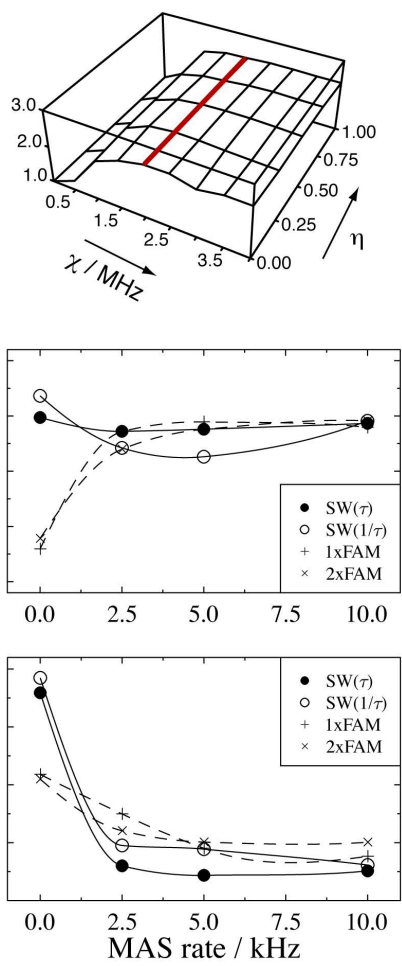

average grid
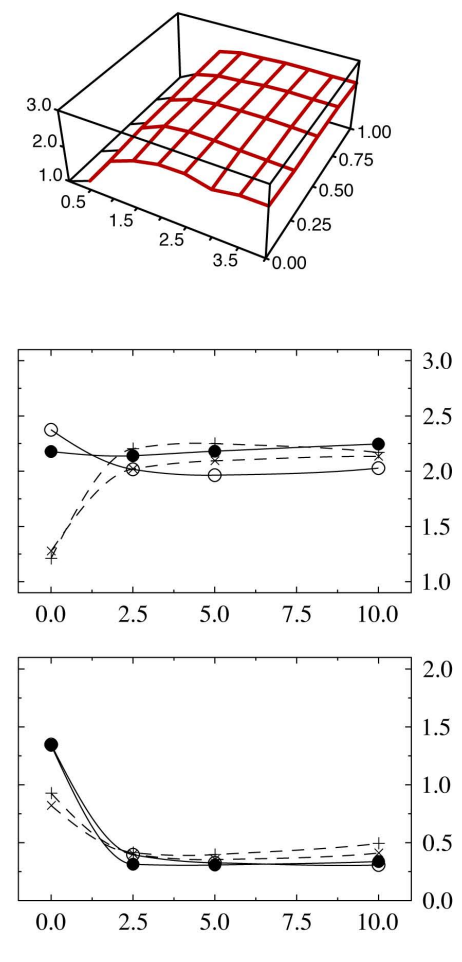

Fig. (6). Evolution of enhancement factors and r.m.s. line shape variation of ${ }^{27} \mathrm{Al}$ central-transition spectra with increasing MAS speed. Signal enhancement by spin-population transfer was generated using single-block FAM-I, double-block FAM-I, SW $(\tau)$-FAM, and SW $(1 / \tau)$-FAM, respectively.

(a) Depiction of the origin of data. The plots in (b) and (c) refer to, respectively: the optimised point with $\chi=2.0 \mathrm{MHz}$ and $\eta=0.5$ only; the average of the line described by $\chi=2.0 \mathrm{MHz}$ and $\eta=0 \ldots 1$; the average of the entire grid, i.e. $\chi=0.5 \ldots 4 \mathrm{MHz}$ and $\eta=0 \ldots 1$.

(b) Evolution of enhancement factor vs. MAS speed. The fitted lines are drawn to guide the eye.

(c) Evolution of r.m.s. line shape variation vs. MAS speed. The fitted lines are drawn to guide the eye.

Finally, we follow the evolution of the spectra when progressively "turning on" the MAS from $0.0 \rightarrow 2.5 \rightarrow 5.0$ $\rightarrow 10.0 \mathrm{kHz}$ spinning speed. In addition to the pulse trains considered above, we now also inspect the perfomance of a FAM-I train with a single modulation frequency. It has been recently demonstrated experimentally in a solid-state NMR study of hydroxyapatites [29], that single-block FAM-I may be sufficient to generate significant signal enhancement under MAS. In our numerical simulations, the best static performance for single-block FAM-I was found for the parameters $\tau_{p}=1.6 \mu \mathrm{s}, N=50$. In Fig. (6), the change of enhancement factors and the r.m.s. line shape variation is plotted for different data sets: only for the point of the grid for which the FAM trains have been optimised $(\chi=2.0 \mathrm{MHz}, \eta$ $=0.5)$, for the average of the $\eta$-line on which the optimised point lies (i.e. $\chi=2.0 \mathrm{MHz}, \eta=0 \ldots 1$ ), and for the average of the entire grid (i.e. $\chi=0.5 \ldots 4.0 \mathrm{MHz}, \eta=0 \ldots 1$ ). From the plot of the enhancement factors (Fig. $(\mathbf{6 b})$ ), it can be seen that the appearance is very similar for all data sets used: The signal amplification obtained from $\mathrm{SW}(1 / \tau)$-FAM drops with the onset of the MAS, to recover again for higher spinning speeds. Nevertheless, its performance is slightly below that of $\mathrm{SW}(\tau)$-FAM and the FAM-I trains. The enhancement gained from applying single-or double-block FAM-I trains is poor under static conditions, but rises sharply with the onset of MAS, to level out for higher spinning speeds. In terms of signal enhancement alone, the advantage of the double-block over single-block FAM-I seems to be limited to static conditions, as under MAS, the single-block FAM-I performs as well or even slightly better. It is however also instructive to look at the evolution of the r.m.s. line shape variations shown in Fig. (6c). From these plots, it can be seen that the additional modulation frequency present for double-block FAM-I benefits the line shape. In extension of this observation, the presence of an entire range of modulation frequencies for $\mathrm{SW}(\tau)$-FAM results in best reproduction of line shape. This effect is especially pronounced in the proximity of the $\{\chi, \eta\}$ pair for which the sequence has been optimised, as can be seen from the left and centre plot of Fig. (6c).

In summary, it may therefore be said that the $\mathrm{SW}(\tau)$-FAM sequence constitutes a good compromise for both static and MAS conditions, as the available enhancement remains almost constant over the investigated MAS speed interval. The evolution of enhancement factors with increasing spinning speed is similar to recently published experimental data [25]. In actual NMR experiments, however, only a limited number of combinations of the quadrupolar parameters $\{\chi, \eta\}$ may be tested, namely those of the compound(s) under investigation. The computational approach described here has the advantage of mapping the performance of the respective FAM trains over an entire 
$\{\chi, \eta\}$ grid. It should be kept in mind that the parameters governing the FAM trains have been only optimised for signal enhancement of the midpoint of the grid $(\chi=2.0 \mathrm{MHz}$, $\eta=0.5)$. In this light, it is remarkable how much the shapes of the curves shown in Fig. (6b) resemble each other for different averaged data sets. This attests to the robustness of the signal enhancement achievable with FAM-trains for a range of $\{\chi, \eta\}$ combinations without the need of further optimisation.

\section{CONCLUSIONS}

Extensive numerical calculations using the Simpson package [27] have been carried out to evaluate and compare the signal enhancing performance of FAM-I, SW $(\tau)$-FAM, and $\mathrm{SW}(1 / \tau)$-FAM sequences under both static and MAS conditions for nuclei with $I=5 / 2$. Numerical results were obtained for a two-dimensional grid of the quadrupolar interaction parameters, namely $\chi=0.5 \ldots 4.0 \mathrm{MHz}, \eta=0 \ldots 1$, and a range of MAS spinning speeds, i.e. $0 \mathrm{kHz}$ (static), 2.5 $\mathrm{kHz}, 5 \mathrm{kHz}$ and $10 \mathrm{kHz}$. In agreement with previously published experimental data $[24,25]$, the best signal enhancement of static spectra is delivered by the $\operatorname{SW}(1 / \tau)$ FAM sequence, whereas under MAS conditions, SW $(\tau)$-FAM and FAM-I trains perform best. Since a FAM-I train consists of a simple sequence of equal RF pulses, it is most easily implemented into the pulse program of a modern NMR spectrometer. On the other hand, if there is interest to acquire spectra under static or slow spinning conditions, it may be worth implementing the slightly more complex $\mathrm{SW}(\tau)$-and/or $\mathrm{SW}(1 / \tau)$-FAM sequences into the pulse programs [30]. It should also be noted that for some systems (such as the ${ }^{47,49} \mathrm{Ti}$ spectra described in Ref. [23]), SW( $\tau$ )-FAM may give slightly better enhancement than FAM-I trains even for MAS spectra. The fact that the total grid average of the signal enhancement shown in both Figs. (4) and (6b) is somewhat higher for $\mathrm{SW}(\tau)$-FAM than for FAM-I may also point in this direction. It has also been demonstrated here that line shapes of the CT spectra are reproduced more faithfully when more than a single modulation frequency is present in the FAM train. Thus, double-block FAM is preferable to single-block FAM, and $\mathrm{SW}(\tau)$-FAM generates the best overall line shapes under MAS conditions.

In summary, the application of spin-population transfer techniques such as the FAM sequences can only be recommended for routine use in solid-state NMR investigations of quadrupolar nuclei with spin $I>1$, as it helps to overcome the sensitivity problems usually associated with these nuclei.

\section{ACKNOWLEDGEMENTS}

Financial support from Deutsche Forschungsgemeinschaft (DFG grant BR 3370/3-1) is gratefully acknowledged.

\section{REFERENCES}

[1] Vega AJ, Quadrupolar nuclei in solids. In: Grant DM, Harris RK, Eds. Encyclopedia of Nuclear Magnetic Resonance. Wiley: New York 1996; vol. 6: pp. 3869-89.

[2] Jerschow A. From nuclear structure to the quadrupolar NMR interaction and high-resolution spectroscopy. Prog NMR Spect 2005; 46: 63-78.

[3] MacKenzie KJD, Smith ME. Multinuclear Solid-State NMR of Inorganic Materials. Pergamon Press: Oxford 2002.
[4] Andrew ER. Magic angle spinning in solid state n.m.r. spectroscopy. Phil Trans R Soc A 1981; 299: 505-20.

[5] Pound RV. Nuclear electric quadrupole interactions in crystals. Phys Rev 1950; 79: 685-702.

[6] Vega S, Naor Y. Triple quantum NMR on spin systems with $I=3 / 2$ in solids. J Chem Phys 1981; 75: 75-86.

[7] Haase J, Conradi MS. Sensitivity enhancement for NMR of the central transition of quadrupolar nuclei. Chem Phys Lett 1993; 209: 287-91.

[8] Haase J, Conradi MS, Grey CP, Vega AJ. Population transfers for NMR of quadrupolar spins in solids. J Magn Reson A 1994; 109: 90-7.

[9] Haase J, Conradi MS, Oldfield E. Single-and double-resonance experiments of quadrupolar nuclei in solids using sensitivity enhancement of the central transition. J Magn Reson A 1994; 109: 210-5.

[10] van Veenendal E, Meier BH, Kentgens APM. Frequency stepped adiabatic passage excitation of half-integer quadrupolar spin systems. Mol Phys 1998; 93: 195-213.

[11] Kentgens APM, Verhagen R. Advantages of double frequency sweeps in static, MAS and MQMAS NMR of spin $I=3 / 2$ nuclei. Chem Phys Lett 1999; 300: 435-43.

[12] Iuga D, Schäfer H, Verhagen R, Kentgens APM. Population and coherence transfer induced by double frequency sweeps in half-integer quadrupolar spin systems. J Magn Reson 2000; 147: 192-209.

[13] Iuga D, Kentgens APM. Influencing the satellite transitions of halfinteger quadrupolar nuclei for the enhancement of magic angle spinning spectra. J Magn Reson 2002; 158: 65-72.

[14] Siegel R, Nakashima TT, Wasylishen RE. Signal enhancement of NMR spectra of half-integer quadrupolar nuclei in solids using hyperbolic secant pulses. Chem Phys Lett 2004; 388: 441-5.

[15] Siegel R, Nakashima TT, Wasylishen RE. Sensitivity enhancement of solid-state NMR spectra of half-integer spin quadrupolar nuclei using hyperbolic secant pulses: Applications to spin-5/2 nuclei. Chem Phys Lett 2006; 421: 529-33.

[16] Siegel R, Nakashima TT, Wasylishen RE. Sensitivity enhancement of NMR spectra of half-integer spin quadrupolar nuclei in solids using hyperbolic secant pulses. J Magn Reson 2007; 184: 85-100.

[17] Yao Z, Kwak HT, Sakellariou D, Emsley L, Grandinetti PJ. Sensitivity enhancement of the central transition NMR signal of quadrupolar nuclei under magic-angle spinning. Chem Phys Lett 2000; 327: 85-90.

[18] Prasad S, Kwak HT, Clark T, Grandinetti PJ. A simple technique for determining nuclear quadrupole coupling constants with RAPT solid-state NMR spectroscopy. J Am Chem Soc 2002; 124: 4964-5.

[19] Kwak HT, Prasad S, Clark T, Grandinetti PJ. Enhancing sensitivity of quadrupolar nuclei in solid-state NMR with multiple rotor assisted population transfers. Solid State Nucl Magn Reson 2003; 24: 71-7.

[20] Madhu PK, Pike KJ, Dupree R, Levitt MH, Smith ME. Modulationaided signal enhancement in the magic angle spinning NMR of spin5/2 nuclei. Chem Phys Lett 2003; 367: 150-6.

[21] Madhu PK, Johannessen OG, Pike KJ, Dupree R, Smith ME, Levitt MH. Application of amplitude-modulated radiofrequency fields to the magic-angle spinning NMR of spin-7/2 nuclei. J Magn Reson 2003; 163: 310-7.

[22] Bräuniger T, Ramaswamy K, Madhu PK. Enhancement of the central-transition signal in static and magic-angle-spinning NMR of quadrupolar nuclei by frequency-swept fast amplitude-modulated pulses. Chem Phys Lett 2004; 383: 403-10.

[23] Bräuniger T, Madhu PK, Pampel A, Reichert D. Application of fast amplitude-modulated pulse trains for signal enhancement in static and magic-angle-spinning ${ }^{47,49}$ Ti-NMR spectra. Solid State Nucl Magn Reson 2004; 26: 114-20.

[24] Bräuniger T, Hempel G, Madhu PK. Fast amplitude-modulated pulse trains with frequency sweep (SW-FAM) in static NMR of half-integer spin quadrupolar nuclei. J Magn Reson 2006; 181: 6878.

[25] Bräuniger T, Madhu PK. Fast amplitude-modulated pulse trains with frequency sweep (SW-FAM) in solid-state NMR of spin-7/2 nuclei. J Magn Reson 2008; 193: 102-9.

[26] Silver MS, Joseph RI, Hoult DI. Selective spin inversion in nuclear magnetic resonance and coherent spin optics through an exact solution of the Bloch-Riccati equation. Phys Rev A 1985; 31: 27535 . 
[27] Bak M, Rasmussen JT, Nielsen NC. SIMPSON: A general simulation program for solid-state NMR spectroscopy. J Magn Reson 2000; 147: 296-330.

[28] Kupce E, Freeman R. Optimized adiabatic pulses for wideband spin inversion. J Magn Reson A 1996; 118: 299-303.

[29] Laurencin D, Wong A, Dupree R, Smith ME. Natural abundance $\mathrm{Ca}$ solid-state NMR characterisation of hydroxyapatite:
Identification of the two calcium sites. Magn Reson Chem 2008; 46 : 347-50.

[30] Supporting information (example pulse programs and a calculation tool) may be downloaded from: http://www.physik.unihalle.de/nmr/ dir tb/swfam.html.

(C) Thomas Bräuniger; Licensee Bentham Open.

This is an open access article licensed under the terms of the Creative Commons Attribution Non-Commercial License (http://creativecommons.org/licenses/by$\mathrm{nc} / 3.0 /$ ), which permits unrestricted, non-commercial use, distribution and reproduction in any medium, provided the work is properly cited. 